\title{
UNIVERSITARIOS Y PRENSA EN GALICIA: HÁBITOS DECLARADOS Y PREFERENCIAS INFORMATIVAS
}

\author{
Carmen Costa Sánchez/ \\ (Universidade da Coruña) \\ carmen.costa@udc.es \\ Miguel Túñez López \\ (Universidade de Santiago de Compostela) \\ miguel.tunez@usc.es
}

\begin{abstract}
Resumen: La presente investigación se inicia con objeto de conocer los hábitos de consumo y adquisición de prensa en papel por parte de los universitarios gallegos. Su interés resulta de especial relevancia, teniendo en cuenta que se trata de un target muy específico (entre 18 y 25 años, en periodo de formación superior), en un contexto mediático de especial interés (despegue de los medios electrónicos, irrupción de la prensa gratuita, programación televisiva de cuestionada calidad, etc.). Los resultados obtenidos permiten realizar una lectura que aporta los datos que definen sus preferencias mediáticas y sus hábitos de consumo informativo.
\end{abstract}

Palabras Clave: Prensa, universitarios, hábitos de consumo, preferencias informativas.

Abstract: The current investigation pretends to know the habits of press consumption and acquisition from the university students in Galicia. His interest is of special significance, considering that this is a very specific target (between 18 and 25 years, university education), in an interesting given media context (boom of electronic media, arrival of free newspapers, TV programming whose quality is criticized, etc. Thanks to the results of this work, we will contribute some clues about their informative habits and preferences.

Keywords: Press, university students, consumption press, informative preferences.

\section{INTRODUCCIÓN Y DISEÑO METODOLÓGICO}

i tenemos en cuenta los datos del Estudio General de Medios (EGM) desde hace diez años hasta la actualidad, la perspectiva respecto del consumo de prensa en España entre la gente joven resulta preocupante, pues desde el año 1996 al 2007 hay cada vez menos jóvenes de edades comprendidas entre los 14 y los 24 años que lean los diarios en papel.

Tabla 1. Evolución del índice de lectura por años.

\begin{tabular}{|c|c|c|c|c|c|c|c|c|c|c|c|c|}
\hline Edad & $\mathbf{1 9 9 6}$ & $\mathbf{1 9 9 7}$ & $\mathbf{1 9 9 8}$ & $\mathbf{1 9 9 9}$ & $\mathbf{2 0 0 0}$ & $\mathbf{2 0 0 1}$ & $\mathbf{2 0 0 2}$ & $\mathbf{2 0 0 3}$ & $\mathbf{2 0 0 4}$ & $\mathbf{2 0 0 5}$ & $\mathbf{2 0 0 6}$ & $\mathbf{2 0 0 7}$ \\
\hline $\mathbf{1 4 - 2 4}$ & 22,0 & 21,0 & 19,3 & 18,5 & 18,1 & 16,4 & 15,7 & 15,7 & 15,1 & 14,4 & 14,3 & 12,8 \\
\hline
\end{tabular}

Fuente: EGM y elaboración propia. 

gratuita en Aragón

Sin embargo, el panorama mediático ha cambiado mucho en diez años y las audiencias jóvenes pueden haberse diversificado. La prensa digital y la llegada de la prensa gratuita son dos acontecimientos que pueden haber afectado al consumo de la prensa tradicional por parte de los jóvenes.

Con todas estas premisas, la lista de preguntas de partida del presente trabajo era amplia: ¿Leen la prensa los jóvenes universitarios de Galicia? ¿La de pago o la gratuita? ¿Prefieren consultar los periódicos electrónicos? ¿Cuáles son los temas que les interesan más? ¿Cuáles menos? ¿Qué opinan de la prensa: es creíble, les parece cara, es entretenida? ¿Que asuntos deberían estar en las páginas de los periódicos y casi no tienen espacio?

Todas estas preguntas se resumen en dos objetivos principales. En primer lugar, conocer en profundidad los hábitos de consumo y las preferencias informativas de los jóvenes universitarios gallegos en relación con la prensa en papel. En segundo,r y como objetivo complementario, indagar en las opiniones y usos declarados de otros medios de comunicación a su alcance (televisión, Internet, etc.).

Para responder a estas preguntas se encuestó a casi mil jóvenes de las tres universidades de Galicia (Universidad de Coruña, Universidad de Santiago y Universidad de Vigo) y de sus siete campus.

Tabla 2. Estudiantes encuestados por campus.

\begin{tabular}{|c|c|c|}
\hline Campus & Facultades & Total de encuestados \\
\hline \multirow{4}{*}{ A Coruña } & Facultad de Arquitectura & 45 \\
\cline { 2 - 3 } & ETSE de Caminos, Canales y Puertos & 42 \\
\cline { 2 - 3 } & Fac. CC. Económicas y Empresariales & 49 \\
\cline { 2 - 3 } & Facultad de Derecho & 60 \\
\hline \multirow{4}{*}{ Vigo } & Facultad de Filología y Traducción & 37 \\
\cline { 2 - 3 } & ETS Ingenieros de Telecomunicaciones & 24 \\
\hline \multirow{4}{*}{ Pontevedra } & Escuela Universitaria de Fisioterapia & 68 \\
\cline { 2 - 3 } de & E.U. de Ingeniería Técnica Forestal & 23 \\
\cline { 2 - 3 } & Escuela Universitaria de Bellas Artes & 44 \\
\cline { 2 - 3 } & Facultad de Ciencias de la Educación & 52 \\
\cline { 2 - 3 } & Facultad de Ciencias de la Comunicación & 81 \\
\cline { 2 - 3 } & Facultad de Psicología & 69 \\
\cline { 2 - 3 } & ETSE (Informática) & 27 \\
\cline { 2 - 3 } & Facultad de Ciencias Políticas & 35 \\
\hline Ferrol & Facultad de Biología & 117 \\
\hline Lugo & Escuela Univ. de Relaciones Laborales & 20 \\
\cline { 2 - 3 } & Facultad de Veterinaria & 37 \\
\cline { 2 - 3 } & Escuela Universitaria de Enfermería & 38 \\
\cline { 2 - 3 } & Facultad de Ciencias & 14 \\
\hline Ourense & Facultad de Humanidades & 5 \\
\hline & Facultad de Historia & 112 \\
\hline
\end{tabular}

Fuente: Elaboración propia. 
Dado que no era factible recorrer todas las facultades de las tres universidades gallegas, realizamos una selección de facultades que respondió a una combinación de dos criterios:

1. Proporcionalidad: De la Universidad de Santiago de Compostela, el número de facultades seleccionadas debía ser superior al de las Universidades de Coruña y Vigo ya que también la Universidad de Santiago tiene una mayor cantidad de alumnos matriculados.

2. Representatividad: Fueron elegidas aquellas facultades que imparten estudios no identificables en ninguna otra facultad de Galicia como pueden ser Bellas Artes en el campus de Pontevedra (Universidad de Vigo), Arquitectura en la de A Coruña o Traducción e Interpretación en Vigo.

El cuestionario diseñado al efecto contaba con una serie de preguntas, divididas en cuatro bloques: Hábitos de lectura; Hábitos de adquisición; Prensa electrónica e Intereses informativos.

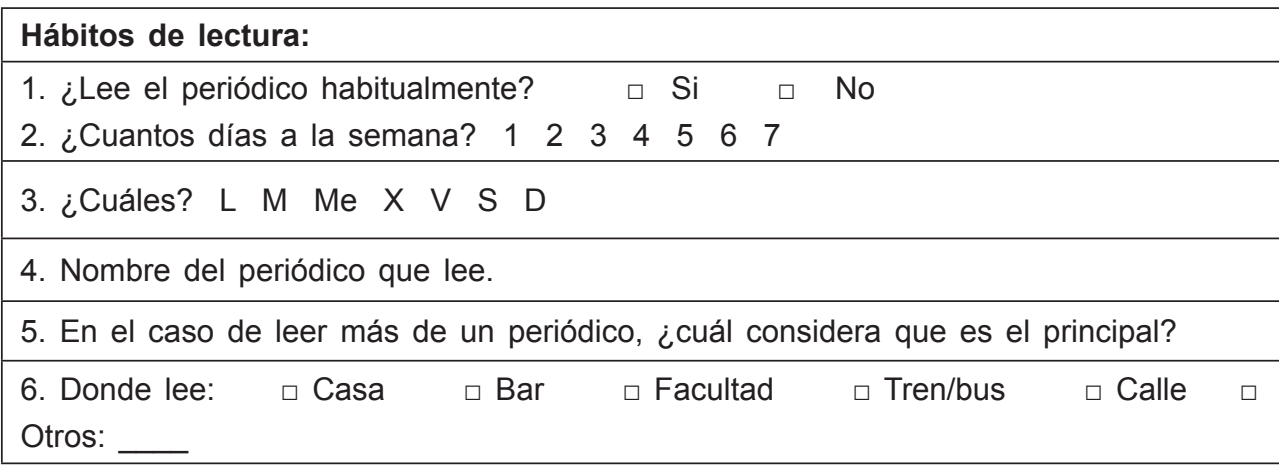

\begin{tabular}{|c|}
\hline Hábitos de adquisición: \\
\hline 7. ¿Compra el periódico habitualmente? $\square \mathrm{Si}$ \\
\hline 8. ¿Cuántos días a la semana? $1 \begin{array}{lllllll}1 & 2 & 3 & 4 & 5 & 6 & 7\end{array}$ \\
\hline 9. ¿Cuáles? L M Me $X \vee S \quad D$ \\
\hline 10. Nombre del periódico que compra. \\
\hline 11. En caso de comprar más de un periódico, ¿cuál considera que es el principal? \\
\hline $\begin{array}{l}\text { 12. Donde compra: } \square \text { Suscripción } \square \text { Quiosco/Tienda } \square \text { Máquina expendendora } \\
\square \text { Otros: }\end{array}$ \\
\hline
\end{tabular}

\section{Nuevas Tecnologías}

13. ¿Consulta algún periódico a través de Internet?

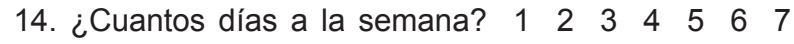

15. ¿Cuáles? L M Me X V S D

16. Nombre del periódico que consulta.

17. En caso de consultar más de un periódico, ¿cuál considera que es el principal? 
Universitarios y prensa en Galicia:

Hábitos declarados y preferencias informativas

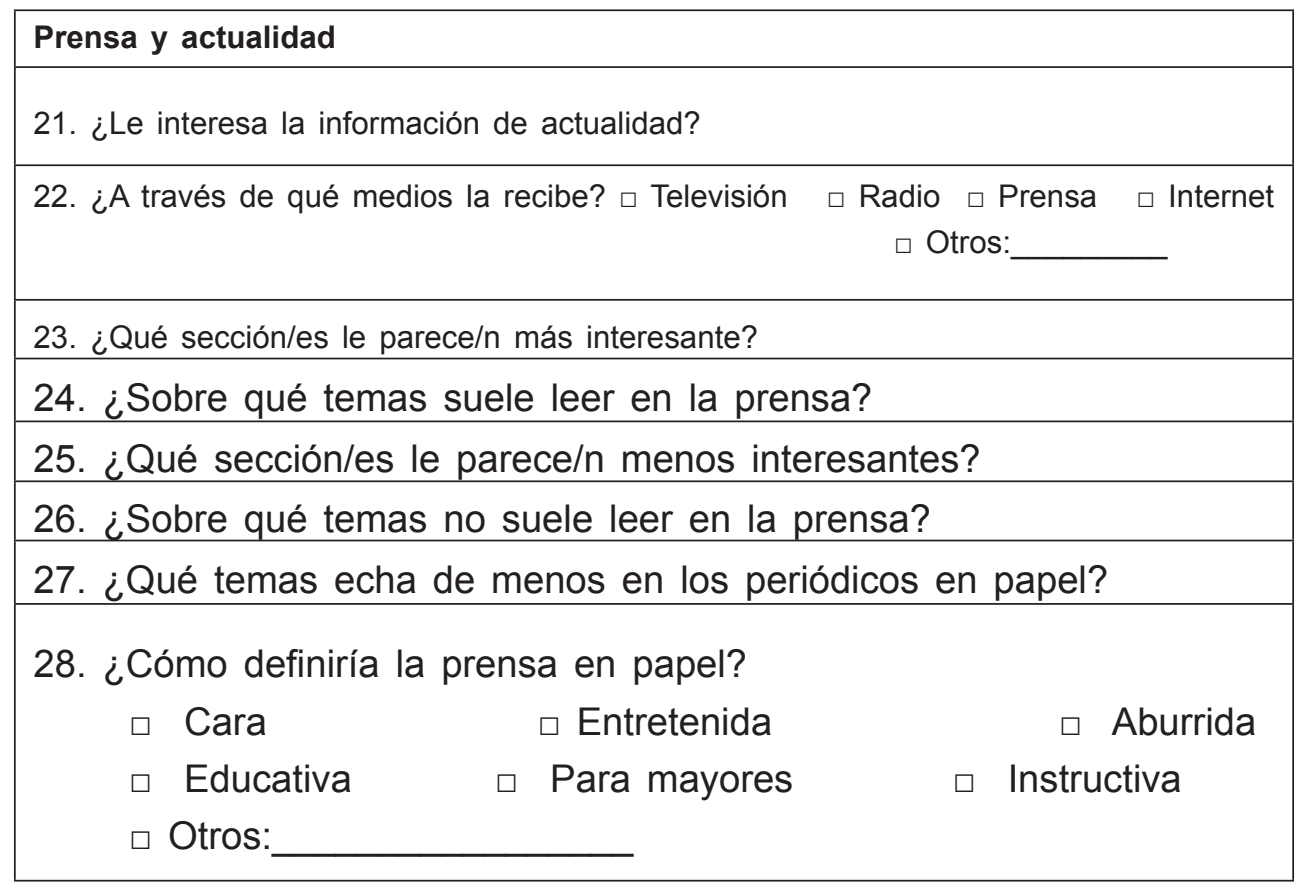

La recogida de datos se realizó de marzo a junio de 2007 tras visitar los siete campus gallegos y las facultades previamente indicadas. El análisis de las respuestas recogidas nos permite poner sobre la mesa los siguientes resultados.

\section{RESULTADOS OBTENIDOS}

\subsection{Hábitos de lectura declarados}

A pesar de que las tres cuartas partes (76\%) de los jóvenes encuestados declaran leer el periódico con asiduidad, el análisis de las respuestas muestra que, en realidad, sólo se pueden considerar lectores de frecuencia alta o muy alta (entre 5 y 7 días de la semana) menos de la mitad (43,6\%). Existe, por lo tanto, la tendencia políticamente correcta de declararse lector habitual de periódicos que no siempre se corresponde con las prácticas de lectura reales. 
Gráfico 1. Frecuencia de lectura de prensa.

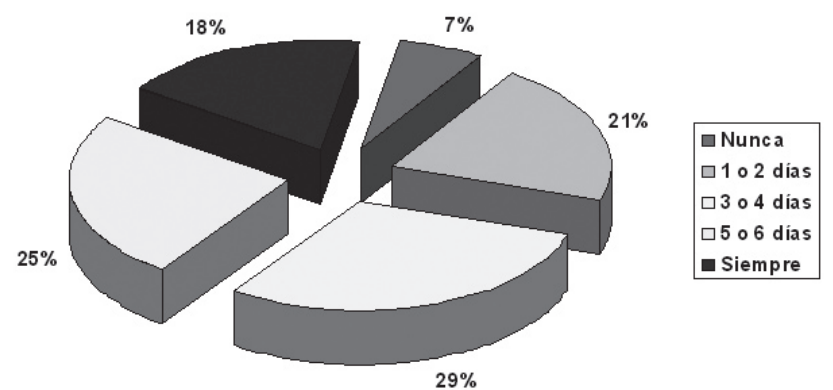

Fuente: Elaboración propia.

\subsection{Ranking de cabeceras más consultadas}

La Voz de Galicia es, con diferencia, la cabecera más consultada por los universitarios gallegos. El segundo puesto, en lo tocante a hábitos de lectura, lo ocuparían los periódicos gratuitos, con un 18\%. En tercer lugar, está El País, periódico que asegura que lee uno de cada ocho encuestados. El cuarto puesto es para Faro de Vigo, consultado por uno de cada diez estudiantes.

De los gratuitos, Metro y 20 Minutos son los que se llevan la mayor parte del porcentaje de lectores con un $33 \%$ y un $29 \%$ respectivamente del total de gratuitos, seguido de lejos por Qué!, $L V$ (De luns a venres) y $A D N$, con un respectivo $19 \%, 11 \%$ y $8 \%$.

\subsection{Ranking de cabeceras de referencia}

Si atendemos a la cabecera considerada de referencia, de aquellos estudiantes que suelen consultar más de una cabecera, tenemos que el periódico más consultado sigue siendo La Voz de Galicia (para un 30\%), seguido por El País $(12 \%)$ y Faro de Vigo (10\%). La prensa gratuita se convierte en la cuarta opción, siendo considerada como tal por un escaso $7 \%$ de los encuestados. La prensa deportiva sólo es considerada como tal por un $2 \%$ del estudiantado.

\subsection{Hábitos de compra}

Seis de cada diez entrevistados (60,3\%) afirma no comprar nunca el periódico y una cuarta parte de los universitarios consultados sólo compra prensa uno o dos días a la semana (22\%). Los que compran la prensa tres o cuatro días a la semana sólo representan el $6,3 \%$ y los que lo hacen cinco o seis días son el 2,6\%. Los que compran todos los días son el $8,7 \%$. Esto es: el número de universitarios que no compra nunca el periódico es casi siete veces superior a los que lo hacen todos los días. 
Gráfico 2. Frecuencia de compra de prensa.

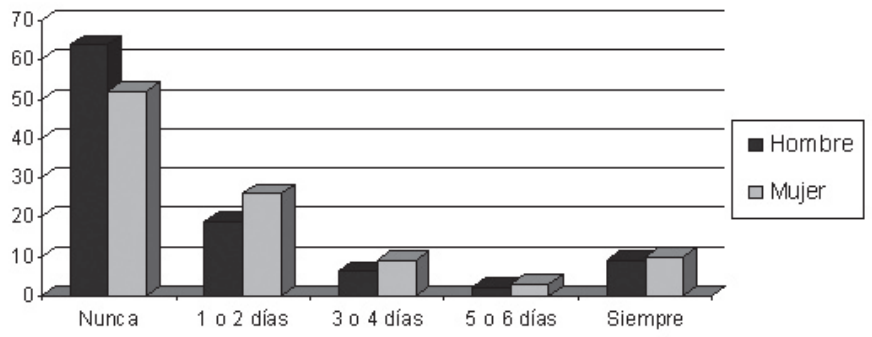

Fuente: Elaboración propia.

\section{5. ¿A través de qué medios se informan?}

Casi todos (un 96,9\%) afirman estar interesados por la información de Actualidad, una información que, según indican, reciben mayoritariamente a través de la Televisión (medio elegido por un $90,5 \%$ de encuestados), seguida por la Prensa (el $77 \%$ de los estudiantes), en tercer lugar la Red $(49,9 \%)$ y, finalmente gracias a la Radio $(43,1 \%)^{1}$.

Gráfico 3. Canal elegido para recibir información.

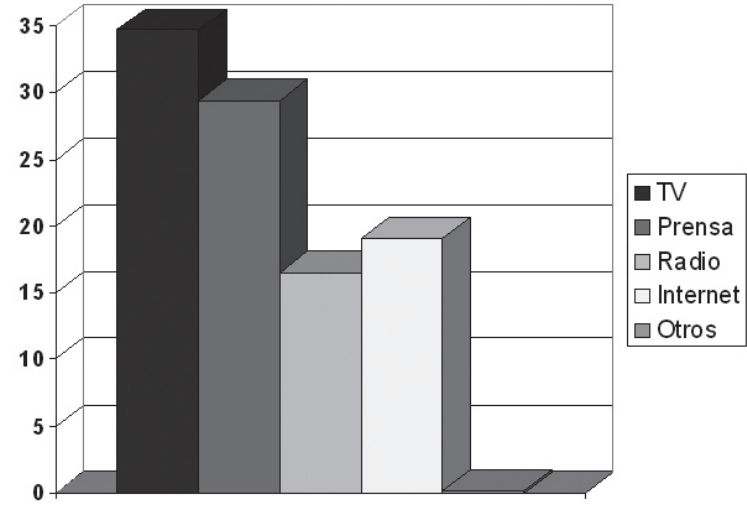

Fuente: Elaboración propia.

\footnotetext{
${ }^{1}$ Las respuestas no eran excluyentes, sino que se trataba de preguntas de respuesta múltiple.
} 


\subsection{Secciones y temáticas de mayor interés}

Deportes, Local, Sociedad, Internacional y Sucesos son, por este orden, las secciones consideradas de mayor interés. Paradójicamente, Deportes es a la vez la más aborrecida, seguida por Economía, Política, Anuncios/Esquelas y Sociedad.

Esta doble elección de Deportes como la sección más y menos atractiva respectivamente se explica por una cuestión de género. Así, del 12,8\% de respuestas que indican Deportes como área de interés, un 22,2\% corresponde a hombres, mientras que un $8,7 \%$ de las mismas corresponde a mujeres. Por el contrario, un $31,8 \%$ de las respuestas de mujeres apuntaba a Deportes como área poco interesante, frente a un $19,7 \%$ de hombres.

Podemos hablar de una polarización de resultados en cuanto a Deportes y en cuanto a Política. Hay a quienes les interesan mucho los Deportes (mayoritariamente hombres) y a quienes no (mayoritariamente mujeres). Hay a quienes les resultan de interés los acontecimientos vinculados al área de Política y a quienes les aburren. Se puede destacar que, según estos datos, son mayoría los que rechazan los temas de Deportes que aquellos a quienes les resultan de interés y que son más los desinteresados en Política que los atraídos por esta sección.

Una tendencia unánime sería el poco interés que despierta en los universitarios los temas de Economía. Estos contenidos no gustan, ni son leídos, por la mayoría de los universitarios gallegos.

Por otra parte, el interés por las denominadas soft news, vinculadas a las áreas de Sociedad y de Cultura, parece confirmarse. Las noticias de proximidad es otra de las líneas temáticas generales por las que los jóvenes sienten curiosidad.

En el caso de los temas de Sucesos vuelve a estar presente la dicotomía de género. Un $11,4 \%$ del total de universitarias encuestadas considera estos temas de interés, frente a un $4,8 \%$ del total de hombres que participaron en la encuesta.

En lo que respecta a los jóvenes que manifiestan una alta frecuencia de lectura de prensa -considerando como tales a aquellos que leen la prensa cuatro días o más a la semana- afirman que la Actualidad, la Cultura, la Tecnología y los Deportes son los temas que más les interesan, por oposición a las secciones de Opinión y Sociedad.

En lo que respecta a los universitarios que afirman comprar el periódico, son la Política, los Deportes y los temas de Sociedad y de Local, por este orden, los que llaman su atención. Por el contrario, aborrecen los de Economía y los Anuncios y Esquelas.

Preguntados por los temas que echan de menos en la prensa, las informaciones de Cultura son las más demandadas por los estudiantes (como indicaron un 18\% de respuestas), así como Ciencia y Tecnología (un 16,1\%), Sociedad y Ocio (12,3\%) o aquéllas que tengan que ver con ellos y sus preocupaciones $(5,1 \%)$. 
Universitarios y prensa en Galicia:

Hábitos declarados y preferencias informativas

En función del área de estudios, se observa la clásica distinción entre la cultura científica y la humanística. Así, los estudiantes de carreras de corte científicotécnico apuestan por incluir más temas de Ciencia y Tecnología (concretamente, los estudiantes de Biología, Telecomunicaciones e Ingeniería de Caminos, por este orden), mientras que estudiantes de Humanidades (de Bellas Artes, Historia y Filología, especialmente) son quienes manifiestan la necesidad de incrementar las informaciones de Cultura.

En función del género, las universitarias piden más: a) Música, Cine y Ocio; b) Cultura; c) Ciencia y Tecnología; d) Juventud. Los universitarios, por su parte, demandan más información sobre: a) Ciencia y Tecnología; b) Música, Cine y Ocio; c) Cultura.

\subsection{Opiniones sobre la prensa en papel}

En el cuestionario se les proponían seis adjetivos (tres positivos y tres negativos), con los que debían calificar la prensa en papel. Las cualidades positivas son resaltadas en mayor número de ocasiones que las negativas. Así, la prensa en papel es definida, primeramente, como Entretenida (39,7\% de respuestas), Instructiva (38,1\%), Educativa (24,4\%), Cara (12,4\%), Aburrida $(11,8 \%)$ y Para Mayores $(8,6 \%)$. Sin embargo, si agrupamos las críticas negativas, un $32,8 \%$ de encuestados creen que es cara, aburrida y para mayores, un porcentaje a tener en cuenta.

\subsection{Prensa electrónica}

En general, el hábito de consulta declarado es bastante alto ya que un $43 \%$ de los encuestados afirman que emplean la Red para informarse.

La tendencia de consulta en Internet viene marcada por la consulta de periódicos de difusión autonómica y nacional y también por los especializados en información deportiva. La Voz de Galicia es el periódico más referenciado entre las cabeceras que los universitarios consultan en Internet $(24,5 \%)$ seguida de El País (22,7\%). Es destacable que casi una sexta parte de los encuestados $(14,8 \%)$ recurran a Internet para consultar Marca, lo que evidencia el interés de los estudiantes gallegos por la información de deportes o, al menos, por el fútbol.

En el caso de los jóvenes que consultan varios periódicos electrónicos, el orden de preferencias se invierte y la mayoría considera El País como el principal de todos los consultados, muy seguido por La Voz de Galicia y en tercera posición, el deportivo Marca.

Dependiendo de las variables demográficas, la consulta de periódicos con importante presencia de lo local adquiere cierta relevancia en función de la población a la que se dirigen. Faro de Vigo, El Progreso, Diario de Arousa o La Región tienen su pequeña cuota de seguidores.

La información deportiva y la política, en el $15,2 \%$ y en el $13,3 \%$ de los casos respectivamente, es la que prefieren los lectores que sólo consultan el periódico en Internet. Las secciones de Deportes y Política, paradójicamente se encuen- 
tran también entre las que menos gustan a los lectores de prensa on line, como ocurría en la prensa en papel. Además, la sección que menos atractivo presenta para los lectores de prensa en Internet es la de Economía, seguida por anuncios y esquelas y por la información rosa.

De igual modo que en el caso de la prensa impresa, se deduce una polarización acusada de las audiencias, de modo que los temas que más leen unos son también los que menos les gustan a otros.

\section{CONCLUSIONES. LA NECESIDAD DE UN CAMBIO DE ESTRATEGIA ORI- ENTADO A LA CAPTACIÓN DE NUEVOS LECTORES}

Entretenida, instructiva y educativa. Los tres calificativos que representan una buena opinión de la prensa en papel recibieron los mayores porcentajes de apoyo frente a las identificaciones de los periódicos como un producto caro, o aburrido o alejado del target ("para mayores" en una muestra de jóvenes). Los resultados también indican que la lectura de prensa es una pauta meritoria en la proyección pública de la conducta social de los individuos, pues hay mayores índices de lectura declarada que de lectura comprobada.

El perfil del universitario en relación con la prensa en papel es el de un lector que consulta el periódico tres o cuatro veces a la semana, pero que no ha adquirido el hábito diario de hacerlo. El lunes despunta como la jornada en que más jóvenes leen el periódico. Este lector tipo se decanta como opción preferente por un periódico regional, La Voz de Galicia, seguida por la prensa gratuita y El País, aunque reconoce calidades y no considera los periódicos gratuitos como prensa de referencia.

Respecto de la adquisición, los datos son claros: sólo el $2 \%$ de los estudiantes puede considerarse comprador habitual de periódicos y un $60 \%$ de ellos reconoce que nunca la compra. A pesar de ello, los universitarios consideran la prensa de pago como la de mayor rigor y calidad, por lo que las cabeceras gratuitas ya no entran en su ranking de los diarios "de referencia".

Sólo uno de cada cuatro reconoce que lee el periódico en casa. Lo común es lo contrario, que ni lo compren ni lo lean en casa. Suelen preferir lugares de uso público en los que, por otra parte, pasan la mayor parte de la jornada. La lista la encabezan los lugares de estudio y formación como las facultades o las escuelas técnicas universitarias donde la cuarta parte de los jóvenes dicen que echan un ojo al periódico durante la jornada lectiva. Bares y cafeterías son la segunda opción escogida.

En una comparativa entre diferentes medios, el acceso a la información se produce a través de una combinación de varios soportes, a pesar de que la televisión se posiciona como el canal más empleado, al ser elegida por nueve de cada diez encuestados. La prensa escrita y la Red son los siguientes en orden de preferencias. 
Universitarios y prensa en Galicia:

Hábitos declarados y preferencias informativas

Al preguntarles por los medios electrónicos, podemos subrayar que Internet se está convirtiendo en un canal habitual de información para los jóvenes y, especialmente, de comunicación y de relación entre ellos. Por el momento, los teléfonos móviles no son soportes habituales empleados para conocer la actualidad. Factores como las tarifas de acceso a Internet de las operadoras, así como aspectos relativos al terminal desde el que se accede (tamaño de pantalla, interfaz de navegación, etc.) son los principales obstáculos apuntados para el bajo empleo del móvil a la hora de informarse (en Bel Mallén, 2010).

En cuanto a las preferencias informativas, existen dos temas que polarizan la opinión de los universitarios: los deportes y la política. En el primer caso se trata, fundamentalmente, de una cuestión de género: a los hombres les interesa mientras que para las mujeres ocupa demasiadas páginas, sobre todo, teniendo en cuenta que el fútbol suele ser el protagonista único de la sección y preferirían que hubiese espacio también para otros deportes. En el caso de la política, hablamos de dos corrientes de opinión opuestas: para una gran parte de los encuestados es un tema imprescindible, mientras que para otros resulta demasiado árido (tal y como demuestra también Farias Batlle, 2007).

En general, todos los estudiantes coinciden a la hora de señalar su interés por las noticias de sociedad, sucesos y cultura, es decir, de las secciones que podemos considerar más "ligeras", frente a las del modelo tradicional (en las que aparecerían grandes áreas como Economía o la ya mencionada Política). A esto hay que añadir la demanda de noticias vinculadas al mundo científico, a las nuevas tecnologías o al medio ambiente, así como una agenda cultural y de ocio más amplia, lo que conduce a pensar en el modelo del periodismo de servicios como más adecuado a sus necesidades.

Dicho modelo se caracteriza por proporcionar información que aporta al receptor la posibilidad de acción y/o reacción. En palabras de Diezhandino (1994: 89):

[...] aquella información, ofrecida oportunamente que pretende ser del interés personal del lector-oyente-espectador; que no se limita a informar sobre sino para; que se impone la exigencia de ser útil en la vida personal del receptor, psicológica o materialmente, mediata o inmediatamente, cualquiera que sea el grado y el alcance de esa utilidad. La información cuya meta deja de ser ofrecer datos circunscritos al acontecimiento, para ofrecer respuestas y orientaciones.

Otros estudios corroboran esta percepción. En este sentido, Arroyo Cabello (2006: 280), en una investigación sobre los hábitos de consumo de prensa de los jóvenes españoles indicaba que:

Los periódicos están cada vez más centrados en el análisis político diario y los contenidos sociales de carácter local están desapareciendo o disminuyendo su peso en la agenda temática. Más o menos esa es la percepción de los jóvenes, que echan de menos más informaciones que "se puedan tocar", es decir, reclaman contenidos de proximidad. 
Esto se ve reafirmado también por el atractivo que suscita la prensa gratuita, a lo que se añade el modo de llegar a los lectores. La prensa gratuita sale a buscarlos a la calle, repartiéndose en mano en lugares y horarios estratégicos, mientras que la prensa de pago hay que ir a buscarla y además pagarla, con lo que requiere una mayor motivación (Sabés, 2005).

La prensa de pago debería, sin embargo, no conformarse con el prestigio del que goza entre los estudiantes universitarios. Debería dedicar esfuerzos a buscar a los lectores jóvenes, adecuando una oferta informativa que les interese y que anime también el hábito de compra. Un periodismo más próximo, más fresco, que anime a la participación del lector puede ser un buen comienzo para que la línea de evolución descendente en las audiencias jóvenes empiece, por fin, su remontada.

\section{REFERENCIAS BIBLIOGRÁFICAS}

ARROYO CABELLO, M.: "Los jóvenes y la prensa: Hábitos de consumo y renovación de contenidos", Revista Ámbitos, n 15 (2006), pp. 271-282.

ALCOCEBA, J. A.; y MATHIEU, G. (2007): Manual de buenas prácticas para el tratamiento de los jóvenes en los medios de comunicación [en línea]. [Consulta: 10 enero 2009]. <http://www.injuve. migualdad.es/injuve/contenidos. .tem. action ?id=2017337609\&menuld=57 2069434>

ASOCIACIÓN PARA LA INVESTIGACIÓN EN MEDIOS DE COMUNICACIÓN (AIMC): Resumen general de resultados EGM [en línea]. [Consulta: 15 diciembre 2008]. <http://www. aimc.es/aimc.php>

BEL MALLÉN, I.: Los jóvenes y la prensa en 2009. En ASOCIACIÓN DE EDITORES DE DIARIOS ESPAÑOLES (2010): Libro blanco de la prensa diaria 2010. Madrid: AEDE, pp. 280-295.

DIEZHANDINO, P. (1994): Periodismo de servicio. Barcelona: Bosch.

FARIAS BATLLE, P. (2007): "Credibilidad de los medios e información política", Jornada de Prensa y Poder Político [en línea]. 2007. [Consulta: 10 enero 2009]. <http://www.funciva. org/uploads/ficheros documentos/1197911338_pedro\%20farias\%20definitivo.doc>

QUESADA, M. (2002): Jóvenes y medios de comunicación. En GARCIA, M.J.; RODRIGUEZ, R; PÉREZ, C.V. (coords.): Cómo mejorar la Imagen de la Juventud en los Medios de Comunicación. Sevilla: Instituto Andaluz de la Juventud, pp. 15-18.

RODRIGUEZ, E. et al. (2001): Jóvenes y medios de comunicación. La comunicación mediática entre los jóvenes madrileños. Madrid: FAD.

RODRIGUEZ, E.; y MEGÍAS, I. (2007): Jóvenes en los medios. Imagen mediática de la juventud, desde su propia mirada. Madrid: FAD.

SABÉS, F.: “Jóvenes y medios: ¿encuentro o desencuentro?", Cuadernos de periodistas, n5 (diciembre, 2005), Asociación de la Prensa de Madrid. 
Universitarios y prensa en Galicia:

Hábitos declarados y preferencias informativas

SALAVERRÍA, R. (2009): ¿Uno para todos y todos para uno? Dimensiones y desafíos de la convergencia periodística. En ASOCIACIÓN DE EDITORES DE DIARIOS ESPAÑOLES: Libro Blanco de la Prensa Diaria 2009. Madrid: Asociación de Editores de Diarios Españoles.

SANCHEZ-TABERNERO, A. (2009): Futuro del periodismo: control de la calidad. En SOUSA, J.P.; y PINTO, R.J. (coords.): IV Jornadas Internacionales de Periodismo Los jóvenes y la renovación del periodismo. Porto (Portugal): Universidad Fernando Pessoa.

SÁNCHEZ, L. et al. (2004): Jóvenes y publicidad. Valores en la comunicación publicitaria para jóvenes. Madrid: FAD.

SANPEDRO, V. (1997): Movimientos sociales: debates sin mordaza. Madrid: Centro de Estudios Constitucionales.

TÚÑEZ, M.: "Jóvenes y prensa en papel en la era Internet. Estudio de hábitos de lectura, criterios de jerarquía de noticias, satisfacción con los contenidos informativos y ausencias temáticas", Estudios sobre el Mensaje Periodístico, no 15 (2009), pp. 503-524.

VV.AA (2008): "Sondeo de opinión y situación de la gente joven. Primera encuesta 2008" [en línea]. 2008. [Consulta: 20 diciembre 2008]. INJUVE. <http://www.injuve.migualdad. es/injuve/contenidos. .tem. action?id=137174936\&menuld $=1008402591>$

\section{Breve semblanza biográfica de los autores}

Miguel Túñez López es Profesor Titular de Producción de la Información en la Universidad de Santiago de Compostela. Miembro del Grupo de Investigación Novos Medios, con numerosas publicaciones sobre newsmaking, comunicación organizacional, comunicación de crisis y actitudes/condiciones laborales de los periodistas.

Carmen Costa Sánchez es Doctora por la Universidad de Santiago de Compostela y profesora en la Facultad de Ciencias de la Comunicación de la Universidade da Coruña, donde imparte las asignaturas de Comunicación e Información Audiovisual y Estructura del Sistema Audiovisual.

(Recibido el 20-02-2009, aceptado el 09-03-2010) 